\title{
Making Sense of Large Datasets in the Context of Complex Situation Understanding
}

\author{
Marielle Mokhtari ${ }^{1}$, Eric Boivin ${ }^{1}$, and Denis Laurendeau ${ }^{2}$ \\ ${ }^{1}$ Defence Research and Development Canada, Quebec City, Canada \\ ${ }^{2}$ Computer Vision and Systems Laboratory, Université Laval, Quebec City, Canada \\ \{Marielle.Mokhtari,Eric.Boivin\}@drdc-rddc.gc.ca, \\ Denis.Laurendeaulagel.ulaval.ca
}

\begin{abstract}
This paper presents exploration prototype tools (combining visualization and human-computer interaction aspects) developed for immersive displays in the context of the IMAGE project. IMAGE supports collaboration of users (i.e. experts, specialists, decision-makers...) for common understanding of complex situations by using a human guided feedback loop involving cutting-edge techniques for knowledge representation, scenario scripting, simulation and exploration of large datasets.
\end{abstract}

\section{Introduction}

Informed decision-making usually results from a proper comprehension of a situation/phenomena. Nowadays, in the military domain, like in many other domains, achieving a good understanding of a complex situation (CS) is a challenge when rapid changes, both operational and technological, occur. This challenge, combined with the objective of increasing the agility of Canadian Forces in dealing with such situations, is investigated in the IMAGE project (Lizotte, Bernier, Mokhtari \& Boivin, 2012). IMAGE aims to support collaboration between users (i.e. experts, specialists, decisionmakers...) to develop a common CS understanding, which can be shared at different levels. The IMAGE concept is supported by four modules: (1) KNOWLEDGE REPRESENTATION, which builds and shares a vocabulary and conceptual graphs making the CS understanding explicit; (2) SCENARIO SCRIPTING, which transforms conceptual graphs into an executable simulation model; (3) SIMULATION (SIM), which exercises the space of scenario variables and browses through simulations; and (4) EXPLORATION (EXP), which exploits visualization and interaction metaphors for investigating large datasets dynamically so data become meaningful to the user. The work presented in this paper focuses on this fourth module, which puts emphasis on the proper use of technologies (and the tools developed for supporting them) in response to the user's exploration needs. Finally, the paper concentrates on non-desktop toolsets developed to help users in making sense of data.

In a wide range of scientific and technological domains (e.g. economics, climate and seismic modeling, melting modeling, astronomy, neuroscience and archaeology), experts need to make sense of and extract useful knowledge from large datasets 
composed of various types of data. To achieve these goals, experts need tools combining visualization and rich user interactions to guide them to incrementally and interactively explore (large) datasets, to organize data, to process information, and to experience and understand these datasets. The EXP module consists in making datasets (generated by the SIM module) explicit in (static and/or dynamic) tailored views, which can be used by users to augment their individual or collective CS comprehension models.

\section{The Exp Concept}

Fig. 1 illustrates the EXP concept, which aims to produce individual or collective explanatory and tailored (static and/or dynamic) views in ways that words cannot communicate clearly. These views, in order to bring a meaning to (raw and/or processed) data, have to generate, stimulate, increase or accelerate the CS understanding. The EXP concept is supported by tools, which implement visualization and interaction approaches by taking advantage of the potential of different platforms in terms of technology and in terms of exploitation of human sensory information (mainly perception and action). The EXP concept has been developed through two main prototypes: the EXP Version 1 (EXP V1) was part of the IMAGE V1 cognitive experiment focusing on individual understanding (Lizotte et al., 2012, sections 7.2 and 8.3) while the EXP Version 2 (EXP V2) was part of the IMAGE V2 feasibility effort and focused on immersive virtual exploration and collaboration aspects (Lizotte et al., 2012, section 7.3).

As represented in Fig. 1, hardware components composing the platforms supporting the EXP tools span from a traditional hardware setup composed of LCD screen / keyboard / mouse to a more high-tech platform exploiting immersive technologies leading to user immersion in a virtual environment (VE) explicating the simulation world initiated by the SIM module. In fact, the EXP module puts emphasis on the proper use of technologies in order to fulfill the user's exploration needs: from (1) conventional non-immersive systems (no immersion, limited interaction), to (2) hybrid systems combining the adequate use of non-immersive systems and immersive interactive systems (in terms of head-centered interaction, access to traditional interaction tools augmented by virtual gadgets) up to (3) high-end fully immersive systems (in terms of large displays, large field of view, head- / hands-centered interaction, more natural interactions). Immersion can be achieved to varying degrees by using advanced human machine interfaces (involving sensory modalities - visual, auditory, tactile, olfactory - and body tracking, etc), and by exploiting more natural interaction with the immersive VE (IVE).

Virtual reality (VR) technology immerses one or more users in a VE representing a real or fictive world that allows users to become agents capable of changing the properties of the environment and interact with its entities (Burdea \& Coiffet, 2003). IVEs, such as CAVEs (Creagh, 2003; Cruz-Neira, Sandin, DeFanti, Kenyon, \& Hart, 1992) support, among other things, the ability to analyze and interpret a large amount of data faster and to explore data in a more intuitive way through interactions with visually rendered data. The CAVE used in IMAGE is a multi-screen projection-based VR system with three walls and a floor that are arranged in a cube for total immersion. 
One of the major advantages of the CAVE is that it makes (collaborative) exploration of large-scale content possible through manipulation and navigation within an IVE. The user navigates the virtual content by naturally moving around inside the cube, while his/her field of view is filled with $3 \mathrm{D}$ representations of components of the environment. To achieve realistic interactions with the CAVE, the tracking system implements fast refresh rates, low latency and smooth tracking. Consequently, it provides smooth and precise estimates of the position and orientation (6 degrees of freedom) of the user's head and hands while not interfering with his/her immersive experience. The tracking system offers ergonomically designed devices: (1) a head tracker attached to stereo glasses; (2) trackers attached to Cybergloves, which are composed of 22 flexible sensors that can accurately measure the position and movement of the fingers and wrist; and (3) a tracked wand, which includes buttons and a joystick for interacting with the virtual content. Stereoscopic rendering based on active glasses provides the user with depth perception of the virtual content.

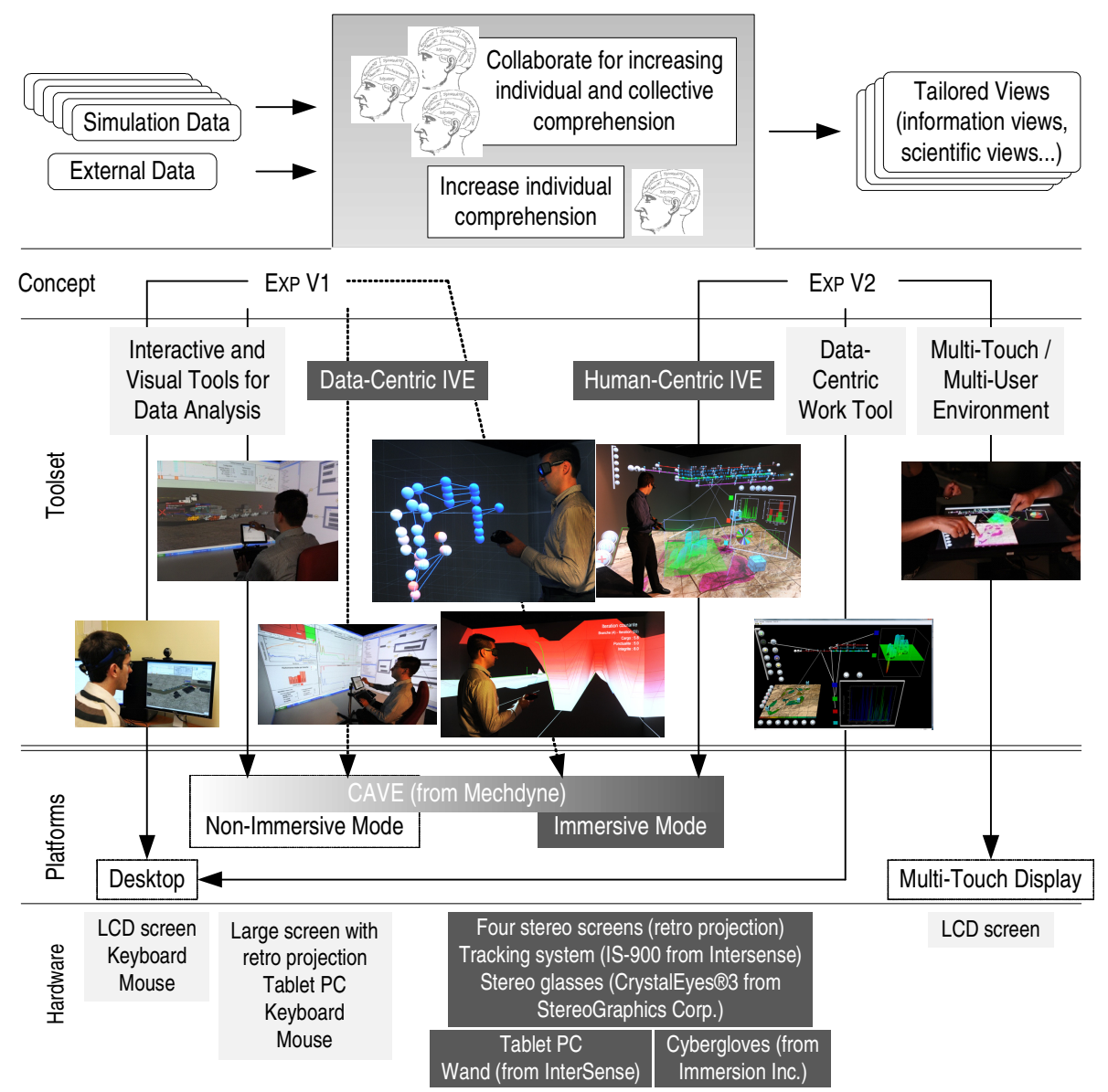

Fig. 1. The EXP module at a glance 


\section{Exp Toolsets in General}

The two versions, EXP V1 and EXP V2, implement interactive and informative simulation analysis tools for exploring different aspects of CSs. To address as much as possible exploration needs with respect to diversity and richness, the toolset proposed in the EXP V1 is a combination of turn-key and commercial off-the-shelf (COTS) tools while the EXP V2 toolset is mainly a custom turn-key tool. The toolsets developed mainly offer the user rapid prototyping of views as well as tailored views and focus on the best view notion rather than the perfect view notion. In the spirit of the Visualization domain, the main task of which is to allow information to be derived from data, the EXP toolsets have been thought to assist users understand and interpret large and rich datasets, by exploiting information rendered graphically (e.g. graphs, diagrams, charts, etc.), which are visual artifacts representing data, illustrating relationships and patterns among data, presenting information (appropriate conversion of data), and also allowing analysis and comparison between graphical representations of datasets. In addition, the EXP toolsets address the dynamic display of trends in the data and human interaction with the displayed data. Consequently the toolsets have been designed to: (1) explore and compare large datasets; (2) extract (hidden) information; (3) discover unexpected trends in the data or find patterns; and, finally, (4) understand specific non-intuitive aspects of CSs. To achieve these goals, the EXP module uses different techniques such as (1) filtering (for focusing on relevant subsets of data) and data brushing (support visual linking of various data and address the visual fragmentation problem of multivariate data representations) (Becker \& Cleveland, 1987), (2) datasets comparison, (3) multi-level datasets exploration, and (4) real-time and stereoscopic rendering.

\section{Exp V1 Toolset - From Desktop Tools to CAVE Tools}

The EXP V1 toolset has been designed to be used on a traditional desktop and on a large screen as well as, at least for a part, on a display wall and in a CAVE, both providing active stereoscopic visualization. Although traditional techniques of data visualization remain conceptually interesting and viable when the user faces a (very) large amount of data to be processed, some techniques become more difficult to implement on a traditional desktop screen. VR allows for the ability to simultaneously analyze a large amount of data / information as well as for a more intuitive exploration in IVEs. The VR gives the impression to the user that he/she interacts and manipulates data directly and not just its graphical representation (Bryson, 1996). Furthermore, multi-dimensional visualization in an immersive context allows users to better understand all the relations between data (Arms, Cook, \& Cruz-Neira, 1999; Raja, Bowman, Lucas, \& North, 2004; Van Dam, Forsberg, Laidlaw, LaViola Jr, \& Simpson, 2000). IVEs provide many benefits for understanding complex systems (Knight \& Munro, 1999), namely the visualization of immersive 3D graphics and different immersion paradigms offering intuitive navigation to explore and analyze data / information. Besides, IVEs facilitate the learning process (Dalgarno \& Lee, 
2010) by, among other factors, promoting the spatial representation of knowledge and augmenting both user's motivation and commitment.

Desktop tools (and parts of) have then been adapted with this in mind so that the user can visualize and explore the data as being "in-the-box i.e. inside looking out" (CAVE version) rather than as being "out-of-the-box i.e. outside looking in" (desktop version). A tablet PC is used to switch between immersive and non-immersive modes, and also to select parameters and data to configure and display. The user can navigate inside the 3D virtual content at will. He/she uses the wand to manipulate (translate, rotate, scale or reset to default values) the 3D graphics. Data selection is not needed because any manipulation involves the whole view. Fig. 2 shows a user manipulating datasets with the wand and visualizing them with the stereo glasses. The data-centric IVEs developed are supported by a custom open architecture, mainly based on openGL and the 3D graphics toolkit OpenSceneGraph (OSG), a 3D graphics rendering engine, used to develop applications in scientific visualization, VR and modeling.

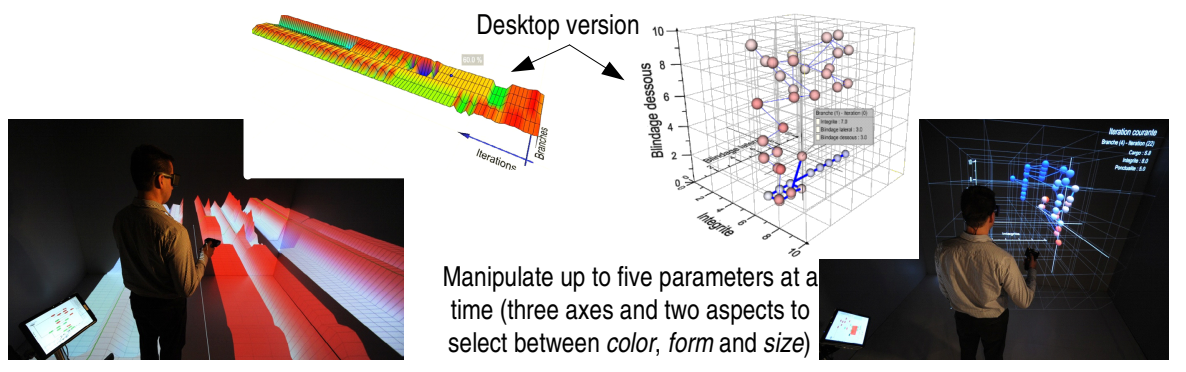

Fig. 2. Exp V1 toolset - From desktop tools to CAVE tools

\section{$5 \quad$ EXP V2 Toolset}

While the development of the EXP V1 toolset has been constrained by cognitive experiments, the EXP V2 is free of all cognitive experiment constraints, hence to the possibility to exploit VR concepts to their full potential and to immerse the user in a dynamic working IVE he/she can build, organize and manage at will by exploiting a toolset for simulation, visualization and analysis. In this second version, the SIM and EXP modules (at least the GUI) are combined within the same environment. A first version of the EXP V2 toolset was presented in (Mokhtari, Boivin, Laurendeau, Comtois, Ouellet, Lévesque \& Ouellet, 2011).

\subsection{Architecture}

The EXP V2 engine is coded in C++ and is based on OSG. The EXP V2 environment can be used on a flat display wall or in a CAVE, both providing active stereoscopic visualization. The architecture also extends to desktop and multi-touch platforms, and supports collaborative work between users. The architecture has been designed to be independent of graphics and physics rendering engines, and is built as a tree, in which 
each branch is an entity, some entities having no correspondence with graphics and physics rendering trees. Three categories of entities have been defined:

1. Manipulator: represents a hardware device (e.g. data glove, wand, keyboard / mouse...) used by human to interact with the objects in the environment. Several Manipulators of the same type can coexist in the EXP V2 environment;

2. User: corresponds to a real user in the environment. It establishes the correspondence between the user and his/her Manipulator(s) and also manages object manipulation to avoid conflict between users;

3. Object: represents an element that is not a Manipulator and with which User can interact. 3D Objects are special instances of Object: their pose (position, rotation, pivoting point and scale factor) can be modified and manipulations (move, rotate, zoom) can be applied on them.

Interaction is considered as a communication mechanism (i.e. flow of messages) allowing Manipulators to send interactions to Objects. Interactions are not directly coupled with Manipulators so the Manipulator design is independent of the Object design. Drag-and-drop is a mechanism that is implemented at the entity level (1) to transfer information from one Object to another (via a ghost concept) and (2) to instantiate associations between two Objects. Another flexible and powerful feature of the architecture is the metadata concept, which encapsulates all data types provided to the EXP module by the SIM module.

\subsection{Fully Immersive Exploration Prototype}

The EXP V2 environment (see Fig. 3) is entirely built, managed and controlled by the user during his/her work session (including object creation, manipulation, destruction, association of objects, etc.). The various objects available, which can be considered as a set of work tools, are presented succinctly in the following subsections (one instance - or several - of the four first work tools is - are - visible on Fig. 3).

Simulation Tree (one scenario, one parameter space). The Simulation Tree is the cornerstone of the EXP V2 environment. It is the direct link between the SIM and EXP modules. The Simulation Tree corresponds to a 3D implementation of the multichronic tree proposed by (Rioux, Bernier \& Laurendeau, 2008), which is the visual representation of the simulation conceptual framework used by IMAGE V1. The Simulation Tree encapsulates both (1) simulations logs in a dynamic layout and (2) simulation data. It can be related to the concept of hierarchy visualization (a branch of Information Visualization) whose visualization and layout techniques have been identified and listed in (Schulz, 2011). Within this classification, the Simulation Tree belongs to the category of 3D dimensionality, an explicit edge (node-link) representation and as exhibiting principal axis-parallel node alignment. Several Simulation Trees can coexist in the EXP V2 environment, each one representing one simulation space created by the user. To mark a point of interest on the Simulation Tree, the user can create sliders, objects that stick to the Simulation Tree and slide along its branches. Combining concepts of data and/or information visualization, the Simulation Tree provides users with (1) a visually 
explicit history of their experiments; (2) a self-explanatory representation of the simulations; and (3) interaction metaphors with running simulations for which parameters and resulting data, rendered in a proper way, can assist users to better understand the simulated CS.

Geospatial View (a means of expliciting a simulation). The Geospatial View allows the simulation status to be visualized as a snapshot at each simulation step (i.e. defined by a slider in the Simulation Tree). The Geospatial View corresponds to a terrain representation on which the scenario elements are displayed symbolically. Information layers and gauges / indicators (being updated at each simulation step) can be added to inform the user of the status of simulation parameters. This tool also permits a simulation to be played (like a video). This object is active if and only if it is associated with a given simulation (in the Simulation Tree) by a drag-and-drop operation.

Scientific Views (comparing simulations in different ways). At any time, to visualize, analyze and explore simulation data or compare simulations, the user can configure Scientific Views (already associated or not to one or several simulations) according to different plot functions: (1) 2D plots such as line (plot), bar (histogram, stacked), and area (pie) graphs. The user can create up to four different graphics within a figure window, each representing different data combinations; and (2) 3D plots that are similar to the 2D plots but in 3D. To associate a simulation to one Scientific View, users can drag and drop this simulation onto a Scientific View and, by a set of menus, select data they wish to visualize and/or analyze (and compare) as well as the type of plot (2D or 3D) to be used. Scientific Views are based on the PLplot library.

Association (communication channel between objects). In order to create an environment in which objects can interact with each other, the concept of association has been implemented at the entity level. This mechanism allows two objects to communicate and exchange data in an asynchronous way using a common protocol. In the environment, an association is represented graphically as a physical link between objects (a bidirectional arrow). Objects' associations can be deleted by the user when they are no longer needed.

Control menus. Two types of control menus are available to users:

1. Main menu (see Fig. 3 and Fig. 4, on the left): is a traditional linear menu composed of a set of icons that represent actions that can be either selected or dragged onto the target object; is always visible; allows to connect the EXP module to the simulator, to create objects (some by changing the value of parameters), and to associate information with elements of the environment. Note: object deletion is achieved by throwing the object above the head of the user (the asymmetric bimanual gestural interface for achieving this operation - and others - is succinctly explained in section 5.3).

2. Circular menu (see Fig. 3 and Fig. 4, on the right): is a hierarchical menu that can be attached to specific objects for additional configuration capabilities (Huot \& 
Lecolinet, 2007); is visible on-demand, and allows selecting which and how data should be visualized and displayed. A circular menu is an interesting technique for displaying many items in a confined space. Besides, it speeds up and optimizes the display options without causing information overload. The item selection time is constant due to the circular organization around the activation point unlike linear menu where the item selection time varies depending on the item position in the menu (Bailly, 2009). Circular menu management is achieved with the asymmetric bimanual gestural interface.

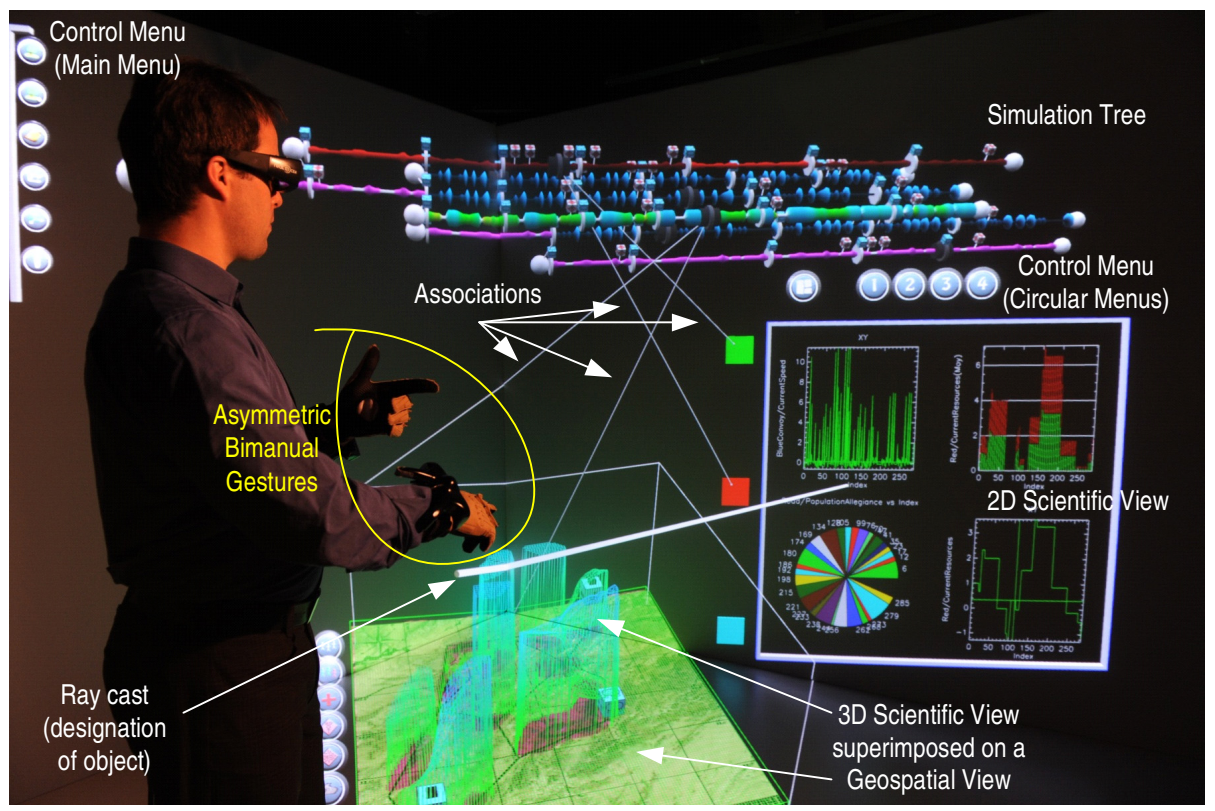

Fig. 3. An instance of the ExP V2 CAVE environment

\subsection{Asymmetric Bimanual Gestural Interface}

To enable the interaction between the user and the IVE, a 3D bimanual gestural interface using Cybergloves has been developed (Lévesque, Laurendeau \& Mokhtari, 2011, 2013). It is built upon past contributions about gestural interfaces and bimanual interactions to create an efficient and intuitive gestural interface tailored to IMAGE needs. Based on real world bimanual interactions, the interface uses the hands in an asymmetric style, with the left hand providing the mode of interaction and the right hand acting at a finer level of detail. The user's actions in the environment have been separated into four categories of gestures: (1) designation (based on ray-casting) and selection of objects; (2) generic manipulations, which group all interactions related to moving / positioning, resizing and rotating objects; (3) specific manipulations, which are interactions tuned for specific objects (e.g. the playback capabilities associated to the Geospatial View - allowing for control of the playback using the right hand's gestures and or pose while the left hand maintains the mode); and (4) system control, 
which represents all actions that are related to (main and circular) menus and modifying the way the environment behaves. Symbolic input of numerical values is also implemented through a behavior similar to that of circular menus, i.e., by rotating the right hand and accepting or cancelling with the left hand. Action examples are presented in Fig. 5. There is no need for travelling interactions since the user is located at the center of the IVE and has access to objects without really navigating in the IVE.

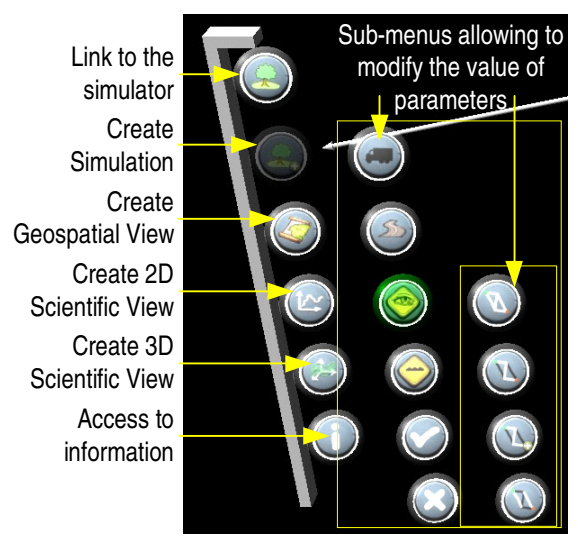

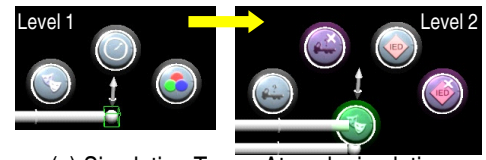

(a) Simulation Tree-At each simulation leaf - Select what data and how to display

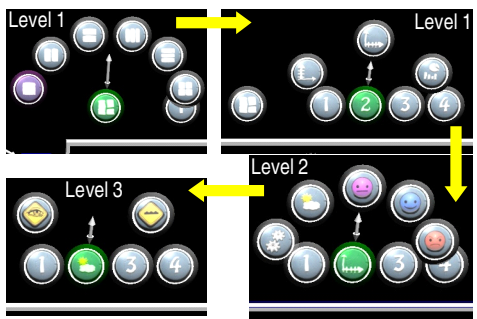

(b) 2D Scientific View - Select the number of graphics, then for each graphic, select what to display in abscissa and in ordinate...

Fig. 4. Control menus of the Exp V2 CAVE environment: main menu + submenus (left) and examples of circular menus + part of their hierarchy (right)

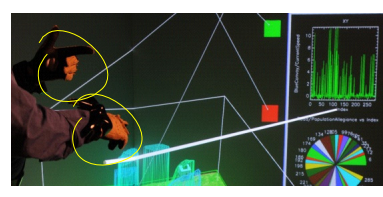

Designation of an object with the left hand and the right hand pointing

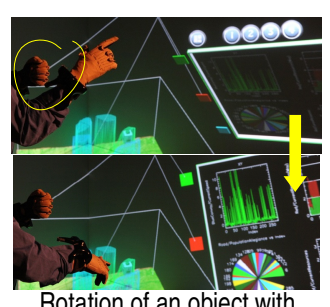

Rotation of an object with the left clenched fist and the right hand pointing

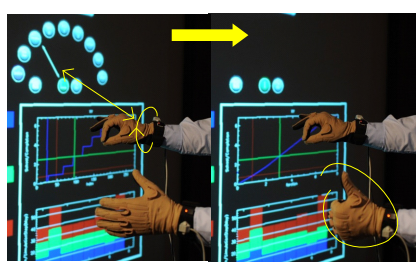

(left) User making a choice in a circular menu - pinch, and (right) user confirming this choice - thumb-up

Fig. 5. Actions: selection (left), generic manipulation (middle), and system control (right)

\section{Conclusion}

In this paper, we introduced different tools that were developed for immersive displays to help human to deal with large datasets composed of different types of data. The EXP V1 tools are complementary tools to turn-key and COTS desktop tools while the EXP V2 toolset is a custom turn-key toolset developed by our team. We are currently exploring ways of connecting several CAVEs in order to implement collaborative work in IVEs in the context of IMAGE. 


\section{References}

1. Arms, L., Cook, D., Cruz-Neira, C.: The Benefits of Statistical Visualization in an Immersive Environment. In: Proc. of Virtual Reality Conference (1999)

2. Bailly, G.: Techniques de menus: Caractérisation, conception et évaluation. Ph. D. thesis (2009) (in French)

3. Becker, R.A., Cleveland, W.S.: Brushing Scatterplots. Technometrics 29(2), 127-142 (1987)

4. Burdea, G.C., Coiffet, P.: Virtual Reality Technology, 2nd edn. Wiley-IEEE Press (2003)

5. Bryson, S.: Virtual Reality in Scientific Visualization. Commun. ACM 39(5), 62-71 (1996)

6. Creagh, H.: CAVE Automatic Virtual Environment. In: Proc. of Electrical Insulation Conference and Electrical Manufacturing Coil Winding Technology Conference (2003)

7. Cruz-Neira, C., Sandin, D.J., DeFanti, T.A., Kenyon, R.V., Hart, J.C.: The CAVE: Audio Visual Experience Automatic Virtual Environment. Commun. ACM 35(6), 64-72 (1992)

8. Dalgarno, B., Lee, M.J.W.: What are the Learning Affordances of 3-D Virtual Environments? British Journal of Educational Technology 41(1), 10-32 (2010)

9. Huot, S., Lecolinet, E.: ArchMenu et ThumbMenu: contrôler son dispositif mobile "sur le pouce". In: Proc. of the 19th International Conference of the Association Francophone d'Interaction Homme-Machine (2007) (in French)

10. Knight, C., Munro, M.: Comprehension with(in) Virtual Environment Visualizations. In: Proc. of the 7th International Workshop on Program Comprehension (1999)

11. Lévesque, J.-C., Laurendeau, D., Mokhtari, M.: Bimanual Gestural Interface for Virtual Environments. In: Proc. of Virtual Reality Conference (2011)

12. Lévesque, J.-C., Laurendeau, D., Mokhtari, M.: An Asymmetric Bimanual Gestural Interface for Immersive Virtual Environments. In: Proc. of the 15th International Conference on Human-Computer Interaction (2013)

13. Lizotte, M., Bernier, F., Mokhtari, M., Boivin, E.: IMAGE Final Report. Technical report 2012-397, DRDC Valcartier (2012)

14. Mokhtari, M., Boivin, E., Laurendeau, D., Comtois, S., Ouellet, D., Lévesque, J.-C., Ouellet, E.: IMAGE - Complex Situation Understanding: An Immersive Concept Development. In: Proc. of Virtual Reality Conference (2011)

15. Raja, D., Bowman, D., Lucas, J., North, C.: Exploring the Benefits of Immersion in Abstract Information Visualization. In: Proc. of the Immersive Projection Technology Workshop (2004)

16. Rioux, F., Bernier, F., Laurendeau, D.: Multichronia - A Generic Visual Interactive Simulation Exploration Framework. In: Proc. of Interservice/Industry Training, Simulation and Education Conference (2008)

17. Schulz, H.-J.: Treevis.net: A Tree Visualization Reference. IEEE Computer Graphics and Applications 31(6), 11-15 (2011)

18. Van Dam, A., Forsberg, A.S., Laidlaw, D.H., LaViola Jr, J.J., Simpson, R.M.: Immersive VR for Scientific Visualization: A Progress Report. IEEE Computer Graphics and Applications 20(6), 26-52 (2000) 